\title{
Proyectos de revitalización de la lengua ulwa'
}

\author{
Esther Núñez Villanueva ${ }^{2}$ y el Comité del Idioma Ulwa (CODIUL) \\ E-mail de contacto: esthernuvi@yahoo.es
}

La desaparición de lenguas indígenas es un fenómeno mundial en esta era globalizada. Se estima que tantas como la mitad de la las lenguas que se hablan en la actualidad habrán desaparecido en el próximo siglo, según Nettle \& Romaine (2000). Estos mismos autores ilustran este proceso analizando la situación en los Estados Unidos, donde antes de la llegada de Colón se hablaban unas 300 lenguas. En la actualidad se hablan solo 175, es decir, aproximadamente la mitad de ellas. Desgraciadamente, la mayoría de estas 175 lenguas indígenas han dejado de ser habladas por los más jóvenes de la comunidad, lo que nos lleva a pensar que desaparecerán en menos de cien años.

En Nicaragua están ocurriendo procesos muy similares. Nettle \& Romaine (2000) han estimado que la recuperación de una lengua es casi imposible cuando quedan menos de 100 hablantes nativos. Podemos enumerar tres lenguas cuyo número de hablantes ha superado o está próximo a esta cifra: la lengua ulwa, la lengua rama y la lengua garífuna, las tres habladas en la Región Autónoma del Atlántico Sur (RAAS).
Según Craig (1992), en los años noventa había alrededor de 40 hablantes de la lengua rama y se estima que en la misma década no había más de una docena de hablantes de la lengua garífuna (Woggon 1992, citado en Koskinen 1995). Aunque se están llevando a cabo esfuerzos de revitalización de estas lenguas en sus respectivas comunidades, es fácil imaginar que no se volverán a hablar en territorio nicaragüense. Mientras la pérdida de la lengua rama es irreparable, ya que se habla únicamente en Nicaragua, la lengua garífuna cuenta con miles de hablantes en otros países como Honduras (Lewis, 2010) $\square$.

El ulwa, por su parte, todavía no ha llegado al límite de 100 hablantes, aunque se acerca peligrosamente a ese número. En el último censo de hablantes realizado en esta comunidad en 1995, se contabilizaron 351 hablantes, la mayoría de ellos de edad adulta (Green \& Hale, 1998). Karawala, a orillas del río Grande de Matagalpa en la RAAS, ha sido identificada como la principal comunidad donde viven los hablantes de ulwa (Véase Mapa 1).

Tabla 1: Hablantes de lenguas amenazadas en la RAAS

\begin{tabular}{|l|l|l|}
\hline Lengua & Hablantes & Censo \\
\hline Rama & 40 aprox. & Craig 1992 \\
\hline Garífuna & 10 aprox. & Koskinen 1995 \\
\hline Ulwa & 351 & Green \& Hale 1998 \\
\hline
\end{tabular}

\footnotetext{
1 Estos proyectos no se podrían haber realizado sin la ayuda del Dr.Andrew Koontz-Garboden, del Instituto de Promoción e Investigación Lingüística y cultural (IPILC) de la Universidad URACCAN de Bluefields, de la Alcaldía de Karawala, del Consejo de Ancianos de Karawala, de la Universidad de Mánchester en el Reino Unido y del Instituto Cervantes de Mánchester en el Reino Unido. A todos ellos les damos nuestro más sincero agradecimiento. Queremos también extender nuestro agradecimiento a los habitantes de Karawala, que participaron en estos proyectos con gran entusiasmo.

2 Esther Núñez Villanueva es una estudiante de la Universidad de Mánchester, Reino Unido, que visitó Karawala apoyada por el Dr. Andrew Koontz-Garborden para colaborar en las actividades del CODIUL de manera voluntaria.
} 
Afortunadamente, el peligro de la lengua ulwa ha hecho reaccionar a la comunidad. Desde finales de los ochenta, Karawala ha entrado en una nueva etapa de desarrollo, lo que ha venido acompañado por un nuevo interés por recuperar la lengua ulwa. En 1988, seis miembros de la comunidad de Karawala fundaron el Comité del Idioma Ulwa (CODIUL), que se traduce en ulwa como Ulwa Yulka Muihka Balna (UYUTMUBAL), con el objetivo de promocionar el uso del ulwa en la comunidad, desarrollar estudios lingüísticos de la lengua y apoyar estudios realizados por personas externas a la comunidad (Hale, 1991) $\square$.

A los seis miembros originales del CODIUL se ha unido más recientemente el profesor don Alberto Santiago William. Los miembros actuales del CODIUL se enumeran en la siguiente Tabla:

\section{Tabla 2: Miembros actuales del CODIUL}

Doña Lorinda Martínez Lacayo

Don Francisco Santiago William

Don Abanel Lacayo Blanco

Doña Clementina Abraham Simón

Don Kandler Santiago Simón

Don Alberto Santiago William

En este artículo se exponen los esfuerzos más recientes que ha llevado a cabo el CODIUL para restaurar el uso de la lengua ulwa en la comunidad durante los pasados meses de mayo, junio y julio de 2009. En primer lugar se ejecutó un proyecto de creación de materiales audiovisuales en ulwa, destinado a ayudar a transmitir la lengua a los más jóvenes. En segundo lugar se realizó un viaje para entablar contactos con otros hablantes ulwa fuera de Karawala. Ambos proyectos contaron con la participación y la ayuda de muchos habitantes e instituciones de Karawala, así como otras institucionales nacionales (universidad URACCAN) e internacionales (Instituto Cervantes de Mánchester y Universidad de Mánchester, Reino Unido). A continuación se detallan ambos proyectos tras una breve introducción sobre la historia del CODIUL y los esfuerzos de revitalización que se han llevado a cabo desde los años ochenta.

\section{Las actividades del CODIUL desde su fundación}

La lengua ulwa puede presumir de su pasado, ya que se ha estimado que era la lengua dominante en un área que se

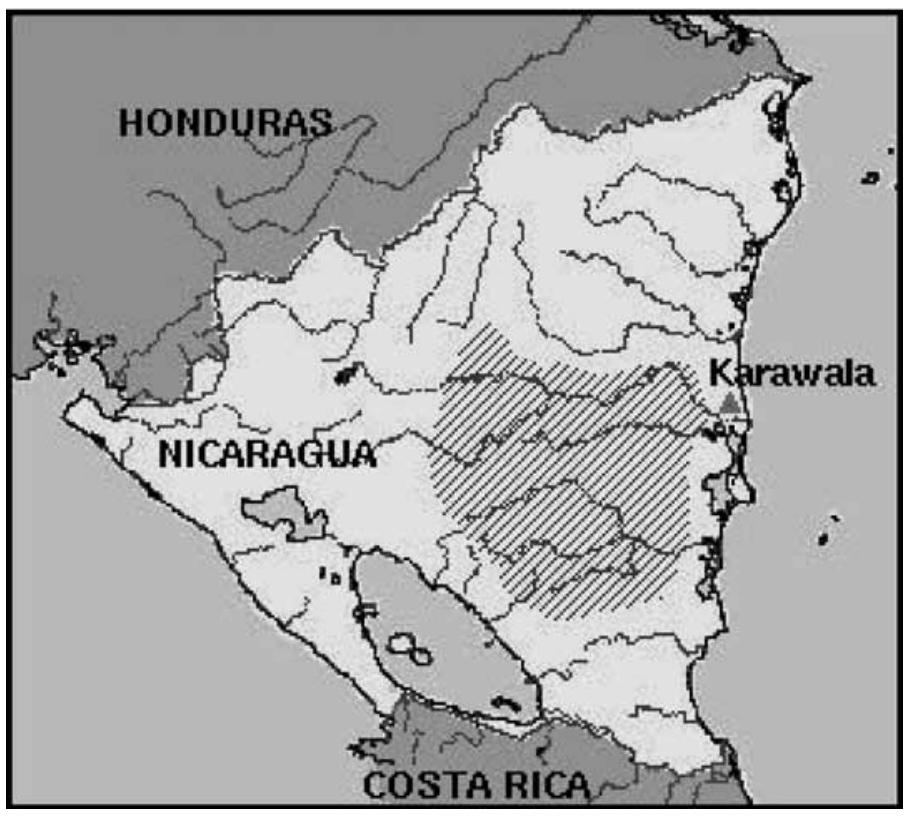

Mapa 1: Situación de Karawala, mapa tomado de slaxicon.org/ulwa

extendía desde las zonas interiores de la RAAS hasta los departamentos actuales de Chontales, Boaco y Matagalpa (Green \& Hale, 1998). Según Green y Hale (1998), hasta 15.000 personas eran hablantes nativos de ulwa en la época pre-colonial. Las persecuciones de los mestizos forzaron a los ulwas a dejar las zonas interiores de Nicaragua para trasladarse a zonas más cercanas a la costa caribe a finales del siglo XIX (Knight \& James, 2008). La tradición oral recogida por los investigadores locales don Leonzo Knight y don Melvin Morton James nos indica que la comunidad de Karawala fue fundada en el año 1850 por seis familias ulwa, tras lo que se convirtió en el principal centro de población ulwa.

Según Green (1999), durante los primeros sesenta años de existencia de Karawala, el ulwa era la lengua predominante de la comunidad aunque la mayoría de la población hablaba bien el miskito. El cambio lingüístico se produjo a partir del año 1950 cuando la compañía norteamericana Nolan estableció un aserradero en la comunidad (Green, 1999). La actividad de la compañía atrajo un gran número de personas de otras etnias en busca de trabajo, de tal forma que el inglés criollo, el español y el miskito pasaron a ser lenguas habituales en la comunidad. Fue el miskito, conocido ya por los habitantes de Karawala, la lengua adoptada como lengua de comunicación y paulatinamente adquirida como lengua nativa por la mayoría de los ulwas nacidos durante o después de la época Nolan (Green, 1999; Knight\& 


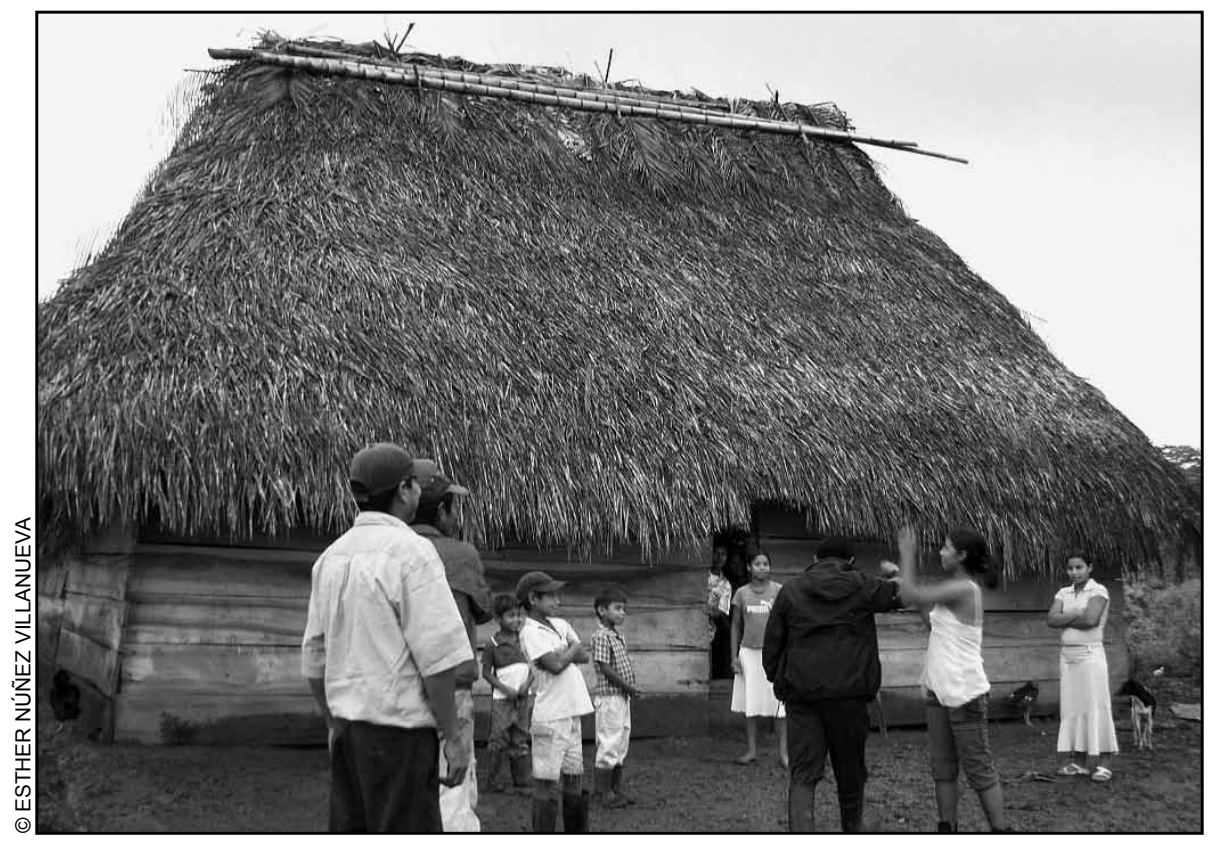

Un momento de la grabación en Tumarín, 2009.

James, 2008). Salazar (2007) describe esta situación como bilingüismo sustractivo, situación en que una lengua pierde terreno frente a otra hasta que termina por desaparecer.

La Ley deAutonomía de 1987 fue el primer documento legal que reconoció los derechos lingüísticos de los nicaragüenses (Koskinen, 1995). Nombró oficiales todas las lenguas habladas en el país, garantizó el derecho a promocionar el uso de dichas lenguas y abrió las puertas a la educación multilingüe (Hale, 2001; Koskinen, 1995). El profesor Francisco Santiago se dio cuenta de que hacía falta realizar un trabajo básico de documentación lingüística del ulwa antes de poder introducirlo en la educación y comunicó esta preocupación a las autoridades regionales competentes. Se consiguió atraer la atención de lingüistas extranjeros tales como Colette Grinevald, colaboradora en el Proyecto de la Lengua Rama, y Ken Hale (Hale, 2001).

Todos estos procesos llevaron al establecimiento del CODIUL en 1988, con el apoyo del académico Ken Hale (Hale, 2001). La labor del CODIUL no ha cesado desde su creación, realizando tanto tareas de documentación lingüística como de promoción cultural y concientización sobre el estado de amenaza del ulwa. Se empezaron a recopilar textos en ulwa y a elaborar los primeros diccionarios durante los años noventa (Knight\& James, 2008). Durante los años 1990 y 1991 se construyó la sede del CODIUL, una pequeña casa de madera que sirve como lugar de reunión, estudio y archivo (Green \& Hale, 1998) ${ }^{3}$.
Más recientemente, dos lingüistas norteamericanos, Tom Green y Andrew Koontz-Garboden han dedicado varios trabajos a la lengua ulwa con la ayuda del CODIUL.

No hay duda de que la medida más importante para revitalizar el uso de la lengua ha sido la introducción de la enseñanza en ulwa gracias al Programa de Educación Intercultural Bilingüe de la Costa Caribe (PEBI). Actualmente, la instrucción exclusivamente en ulwa se realiza durante los primeros cuatro años de la educación primaria (Salazar, 2007). El estudio sociolingüístico realizado por la profesora Orintia Salazar nos informa que alrededor de un $81 \%$ de los niños de 4-5 años, que iniciaron su educación en ulwa, consideran el ulwa su lengua materna, lo que contrasta con el porcentaje, entre $50 \%$ y $58 \%$, de los mayores de cinco años, que han estudiado en miskito. La profesora Salazar identifica correctamente que los encuestados confunden su identidad étnica con su lengua materna, de ahí que ese porcentaje no se corresponda con el número de niños que hablan ulwa de forma fluida. Sin embargo, el aumento de ese porcentaje es una indicación del mayor prestigio que ha alcanzado la lengua y del renovado interés por aprenderla.

En la Tabla 3 se resumen los proyectos realizados por el CODIUL desde su fundación y los proyectos futuros, para los que se necesita el apoyo económico de instituciones regionales o internacionales. Los dos proyectos resaltados se describen en las siguientes secciones.

\footnotetext{
3 Según las últimas noticias, la casa ulwa sufrió graves daños durante el huracán Ida del pasado mes de noviembre de 2009. Además de los daños estructurales a la casa es posible que se hayan perdido textos y documentos fruto del trabajo del CODIUL durante los últimos veinte años.
} 
Tabla 3: Actividades más relevantes del CODIUL desde su fundación

\begin{tabular}{|c|c|c|}
\hline Proyectos & Realizados & Planeados \\
\hline $\begin{array}{l}\text { Trabajos } \\
\text { lexicográficos }\end{array}$ & $\begin{array}{l}\text { - Diccionario preliminar del ulwa (CODIUL/ } \\
\text { UYUTMUBAL, CIDCA, \& MIT, 1996). } \\
\text { - Creación y estandarización de neologismos. }\end{array}$ & $\begin{array}{l}\text { - Revisión del diccionario preliminar. } \\
\text { - Creación de un diccionario infantil. } \\
\text { - Creación de un diccionario de términos } \\
\text { lingüísticos. }\end{array}$ \\
\hline $\begin{array}{l}\text { Trabajos de } \\
\text { investigación } \\
\text { lingüística }\end{array}$ & $\begin{array}{l}\text { - Tesis doctoral de Tom Green (estudio } \\
\text { lexicográfico y gramática). } \\
\text { - Estudios lingüísticos de Don Andrew Koontz- } \\
\text { Garboden (Koontz-Garboden 2007; 2009). } \\
\text { - Monografía de Doña Orintia Salazar para la } \\
\text { Licenciatura en Educación Intercultural Bilingüe } \\
\text { en URACCAN (Salazar 2007). }\end{array}$ & $\begin{array}{l}\text { - Elaboración de una gramática pedagógica de } \\
\text { ulwa. }\end{array}$ \\
\hline Educación & $\begin{array}{l}\text { - Introducción del ulwa hasta el cuarto grado de } \\
\text { primaria. } \\
\text { - Recopilación de textos sobre la comunidad. } \\
\text { - Creación de materiales audiovisuales. }\end{array}$ & $\begin{array}{l}\text { - Introducción de la enseñanza del ulwa en } \\
\text { secundaria. } \\
\text { - Elaboración de materiales didácticos. } \\
\text { - Evaluación de los resultados de la } \\
\text { introducción del ulwa en primaria. }\end{array}$ \\
\hline $\begin{array}{l}\text { Trabajos de } \\
\text { promoción } \\
\text { cultural }\end{array}$ & $\begin{array}{l}\text { - Organización del día del pueblo ulwa, que se } \\
\text { celebra cada } 6 \text { de mayo. } \\
\text { - Publicación de: } \\
\text { Wâlangwas, Historia oral de la comunidad de } \\
\text { Karawala (Knight\& James, 2008). } \\
\text { El pueblo Ulwa: identidad y ambiente en un } \\
\text { contexto multiétnico. (Koskinen (ed.) 2007) } \\
\text {-Encuentro con hablantes ulwa fuera de la } \\
\text { comunidad. }\end{array}$ & $\begin{array}{l}\text { - Recuperación del mapa histórico donde se } \\
\text { establece el límite del territorio ulwa (Tratado } \\
\text { Harrison-Altamirano de 1905). }\end{array}$ \\
\hline Infraestructuras & - Construcción de la casa ulwa. & - Mantenimiento de la casa ulwa. \\
\hline Otros & & $\begin{array}{l}\text { - Elaboración de un censo del total de } \\
\text { hablantes, dentro y fuera de Karawala. } \\
\text { - Promoción del uso del ulwa en las iglesias, } \\
\text { en la clínica, en la alcaldía, en la casa de } \\
\text { justicia y en las ventas. Por ejemplo, mediante } \\
\text { la traducción de carteles y de textos religiosos. }\end{array}$ \\
\hline
\end{tabular}




\section{Proyecto de creación de materiales audiovisuales}

En discusiones con el CODIUL se pensó que sería útil crear una serie de DVDs grabados en ulwa con la participación de la comunidad. El material audiovisual cuenta con varias ventajas importantes:

a) se puede reproducir fácilmente y a bajo costo, por lo que es fácil distribuir copias de los DVDs entre la comunidad y las instituciones interesadas, tales como escuelas, las universidades, etcétera.

b) son materiales más atractivos para las generaciones más jóvenes, que van a tener un mayor interés en ver el DVD en la televisión que en leer un libro.

c) constituye un material lingüístico de gran valor para futuras investigaciones lingüísticas.

El proyecto tuvo resultados excelentes. En dos meses recolectamos más de ocho horas de video. Grabamos a más de veinte personas que se documentaron concienzudamente para hablar de temas muy variados relacionados con Karawala, tales como:

- La historia de Karawala

- La historia y funcionamiento de las diferentes iglesias

- La creación de nuevas zonas de viviendas

- El funcionamiento de las escuelas primaria y secundaria

Parte del proyecto era también realizar grabaciones entre los estudiantes de Karawala. Se pensó que los niños podrían contar historias, entrevistar a sus familiares e incluso representar una obra de teatro para grabarla. Por desgracia, este proyecto no se pudo realizar por varias razones, entre las que se pueden destacar la vergüenza de los jóvenes para participar en este tipo de tareas o el coincidir las grabaciones con exámenes y otras tareas escolares. Queda, por tanto, este proyecto pendiente.

En la actualidad se está procediendo a editar las grabaciones y a crear los DVDs en el Reino Unido. Tras la edición de las grabaciones se procederá a grabar los DVDs y a enviarlos a Karawala y otras instituciones regionales, como las universidades costeñas.

\section{Proyecto de encuentro con hablantes ulwa fuera de la comunidad}

Anteriormente se ha mencionado que seis familias llegaron por el río Grande de Matagalpa, a finales del siglo XIX, a donde hoy se encuentra Karawala (Knight \& James, 2008). Si esto es así es posible que haya otras familias ulwa en otras zonas de Nicaragua que no emigraron a Karawala. Algunos encuentros casuales con estos ulwas en centros como Bluefields confirman esta teoría, pero el CODIUL estima que es necesario realizar un estudio más sistemático de las comunidades donde todavía hay hablantes ulwa.

El contacto con estos hablantes ulwa es necesario por varias razones. En primer lugar, establecer lazos de solidaridad y cooperación entre los ulwa es un deseo natural de la comunidad. En segundo lugar, desde el punto de vista histórico, delinear los lugares donde se habla o se ha hablado ulwa nos permite establecer la zona originaria de los ulwa, así como identificar los lugares en los cuales se fueron estableciendo durante el siglo XIX. Es posible también que esos hablantes recuerden otros aspectos de la historia del pueblo ulwa que son desconocidos en Karawala.

Por último, desde el punto de vista lingüístico, estos contactos pueden ser fundamentales. Nos permiten establecer el número total de hablantes ulwa, un dato a tener en cuenta a la hora de evaluar el estado de amenaza de la lengua. El análisis de otras variedades ulwa también nos puede abrir una puerta a la evolución de la lengua en los últimos años, en especial permitiría establecer si el ulwa de Karawala ha sufrido cambios importantes por el extenso contacto con la lengua miskita. Además, sería necesario considerar estas comunidades a la hora de implementar el Programa de Educación Intercultural Bilingüe.

Teniendo esto cuenta pusimos en marcha el primer encuentro con hablantes ulwa en otras comunidades el pasado mes de julio de 2009. Según la disponibilidad de los miembros del CODIUL y de la financiación que pudimos conseguir, planeamos un viaje de tres días a lo largo del río Grande incluyendo visitas a las comunidades de Guadalupe, Makantakita, Walpa Dâkura y Tumarín. Véase el mapa 2, donde se ilustra el recorrido. 


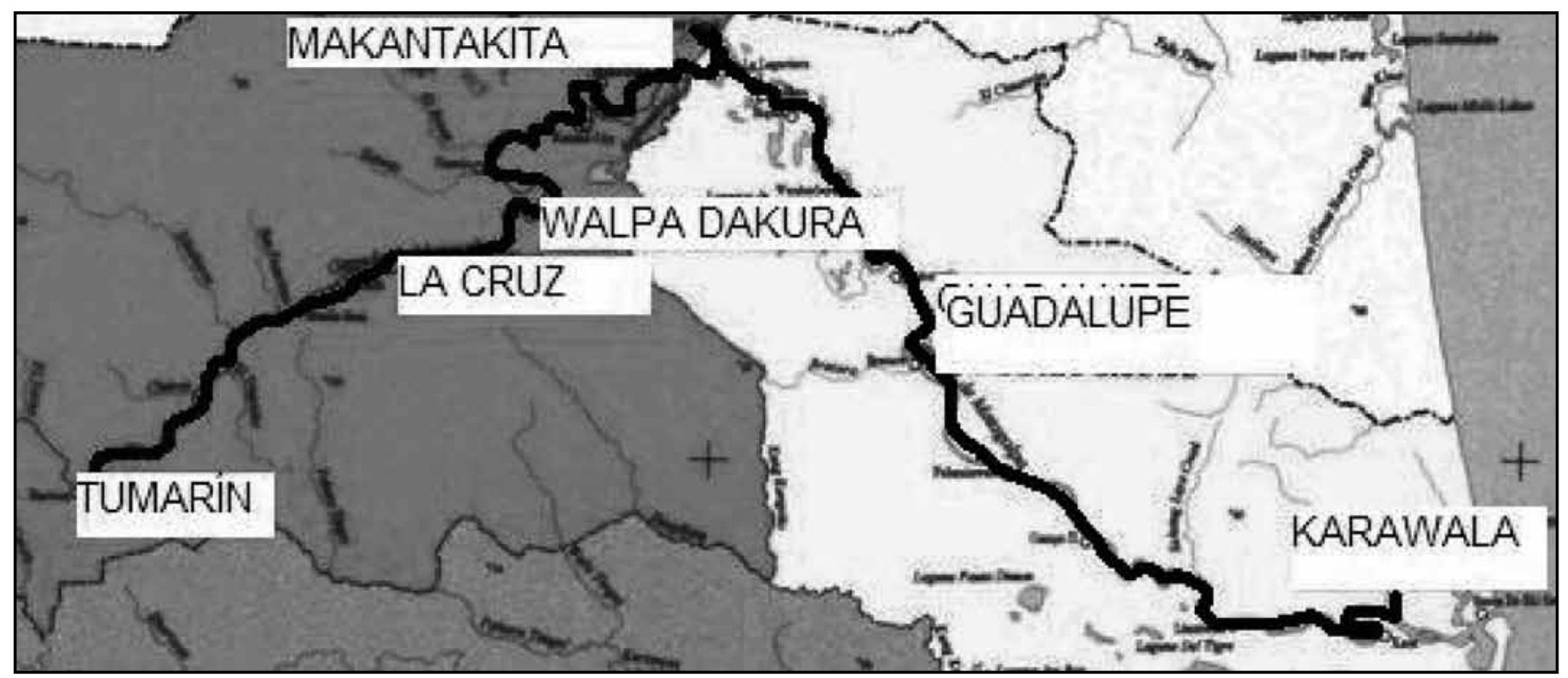

Mapa 2: Recorrido del viaje por el río Grande de Matagalpa.

\subsection{Participantes}

Participaron en la expedición el profesor Don Francisco Santiago, el profesor Don Alberto Santiago, el profesor Don Luis Martinez, la señorita Kenya Wilson y Doña Esther Núñez Villanueva, estudiante visitante de la Universidad de Mánchester. Los dos primeros son miembros activos del CODIUL, mientras que el profesor Luis Martínez es el vicedirector de la escuela primaria de Karawala. La señorita Kenya Wilson, estudiante del Instituto de secundaria de Karawala, vino para establecer contactos con los miembros más jóvenes de las familias que visitamos. Los otros miembros del CODIUL tenían algún otro compromiso para la fecha del viaje o estaban demasiado mayores para participar en este tipo de iniciativas.

\subsection{Financiación}

Para realizar el viaje era necesario alquilar una panga, pagar un panguero y costear la alimentación de los participantes. Agradecemos el apoyo primordial de las instituciones locales. La Alcaldía de Karawala se hizo cargo del salario del panguero mientras que la panga fue provista por Doña Carmela Baptis y Don Silvio Simon. El Consejo de Ancianos nos donó 400 córdobas para los gastos de alimentación.

Los profesores del Instituto Cervantes de Mánchester,
Reino Unido, aportaron 167 dólares (3.300 córdobas) para subsanar el costo de la gasolina. El Instituto de Promoción e Investigación Lingüística y cultural (IPILC), perteneciente a la universidad URACCAN de Bluefields, contribuyó también con fondos para subsanar el costo de la gasolina.

\subsection{Evaluación de los objetivos}

\subsubsection{Objetivo 1: Investigación lingüística.}

Cuantificar el número de hablantes de ulwa en otras comunidades aparte de Karawala y estimar la fluidez de dichos hablantes en ulwa y la vitalidad de la lengua ${ }^{4}$ :

- Guadalupe: En Guadalupe visitamos a la familia de una señora ulwa originaria de Mahogany Creek. De su familia, la mayoría de los hijos entienden pero no hablan ulwa. Solo dos de los hijos hablan ulwa con fluidez, aunque a veces utilizan palabras en miskito o español. La lengua ulwa está en vías de dejarse de hablar en la comunidad de Guadalupe, ya que la lengua de comunicación con los niños es el español. Esta familia manifestó interés en tener educación en ulwa. Se consideran ulwa y no quieren olvidar su lengua.

- Makantakita/Tumarín: Visitamos a una familia originaria de Tumarín, de donde salieron para Rosita durante la década de los 80 a causa de la guerra.

\footnotetext{
4 No hemos incluido el nombre de las personas entrevistadas para preservar su anonimato. En la casa del idioma ulwa han quedado archivados informes y fotografias del viaje. Alli quedan a disposición para cualquier investigador interesado. También, los interesados se pueden poner en contacto con nosotrosen el e-mail esthernuvi@yahoo.es
} 
Vivieron veinte años en Rosita y posteriormente se trasladaron a Makantakita, donde han vivido cuatro años. Toda la familia habla ulwa: los dos abuelos, los seis hijos de ellos y los nietos, es decir, unos 22 hablantes de ulwa. Uno de los hijos de esta familia vive en Tumarín con su familia. La lengua de comunicación de la familia es ulwa y los niños más pequeños la están aprendiendo, por lo que se está transmitiendo a las generaciones más jóvenes.

- Walpa Dâkura: Visitamos a un señor ulwa originario de Tumarín quien habla bien ulwa, pero a veces acusa la falta de uso de la lengua. Ninguno de sus hijos o nietos habla ulwa.

Todas las familias accedieron a ser entrevistadas por los miembros del CODIUL. En total realizamos casi tres horas de grabación en estas comunidades. La participación de estas familias se incluirá en los DVDs.

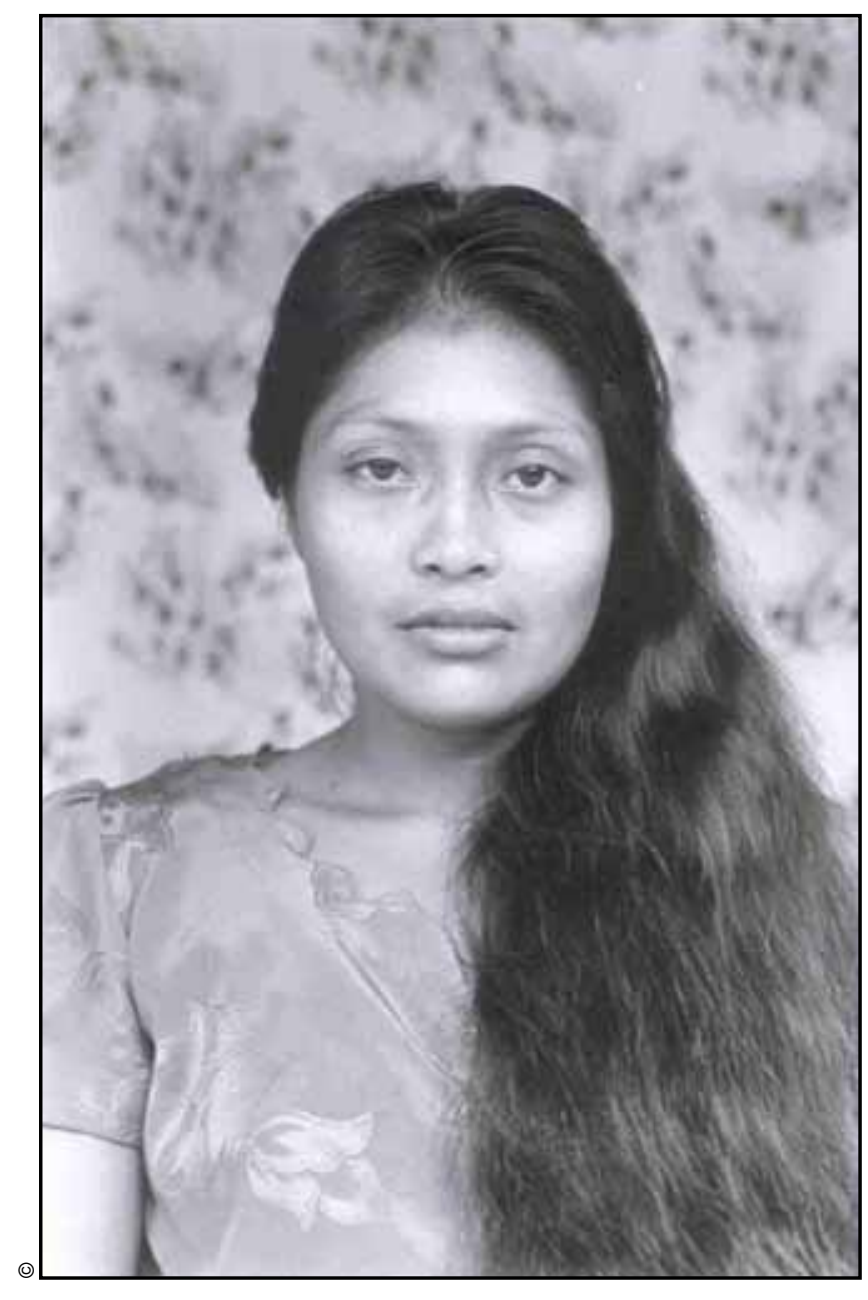

En conclusión, solo la familia de Makantakita ha mantenido el ulwa como lengua de comunicación de la familia. Sería importante mantener los contactos con esta familia, para proporcionar educación en ulwa a los niños de la familia y para realizar estudios de interés lingüístico.

\subsubsection{Objetivo 2: Investigación histórica. Recolección de historias orales.}

Los entrevistados fueron reacios a contar historias. Posiblemente les dimos poco tiempo para su preparación, ya que por razones de tiempo solo pudimos pasar un par de horas con cada familia. La experiencia demostró que, transcurrido más tiempo, la comunicación entre los participantes se hacía más fluida.

Uno de los miembros de la familia de Makantakita nos contó dos historias escuchadas a su abuelo, que en algunos aspectos coinciden con historias contadas en Karawala. En primer lugar, nos relató la historia de un hombre a quien llamaban "Pan Pih", quien tras matar a su esposa escapó varias veces de la policía saltando desde la panga donde lo llevaban apresado a Bluefields. Se dice de dicho individuo que podía estar varias horas bajo el agua. La habilidad de los ulwa para nadar bajo el agua durante largos periodos de tiempo también se cuenta en Karawala, donde se dice que Crimmins, uno de los fundadores de Karawala, podía estar bajo el agua sin ayuda durante mucho tiempo (Knight $\&$ James, 2008). Asimismo, entre su familia se cuenta que antiguamente las familias ulwa compartían viandas durante la navidad, creencia que se cuenta también en Karawala.

Los datos recogidos apuntan a que hubo al menos dos migraciones de ulwas, una a lo largo del río Grande y otra a lo largo del río Mico. Tumarín, de donde proceden la familia residente en Walpa Dâkura y la familia de Makantakita, parece haber sido un centro ulwa importante a orillas del Río Grande. Es posible que los ulwa de Karawala estén relacionados con los ulwa de Tumarín, lo que explicaría la coincidencia de las historias orales. Según nos informaron, las familias ulwa de Tumarín se fueron en la época de la guerra para Rosita. Algunos volvieron a Tumarín, aunque volvieron a trasladarse posteriormente a otras comunidades como Makantakita o Karawala. Es posible que algunas de estas familias continúen viviendo en Rosita.

Green (1999) nos informa que otra investigadora francesa, de nombre Palombieri, documentó en 1967 el dato que se había producido una migración de ulwas desde el río Mico hasta Mahogany Creek en una época similar a la migración que fundó Karawala, es decir, a finales del siglo XIX. Es 


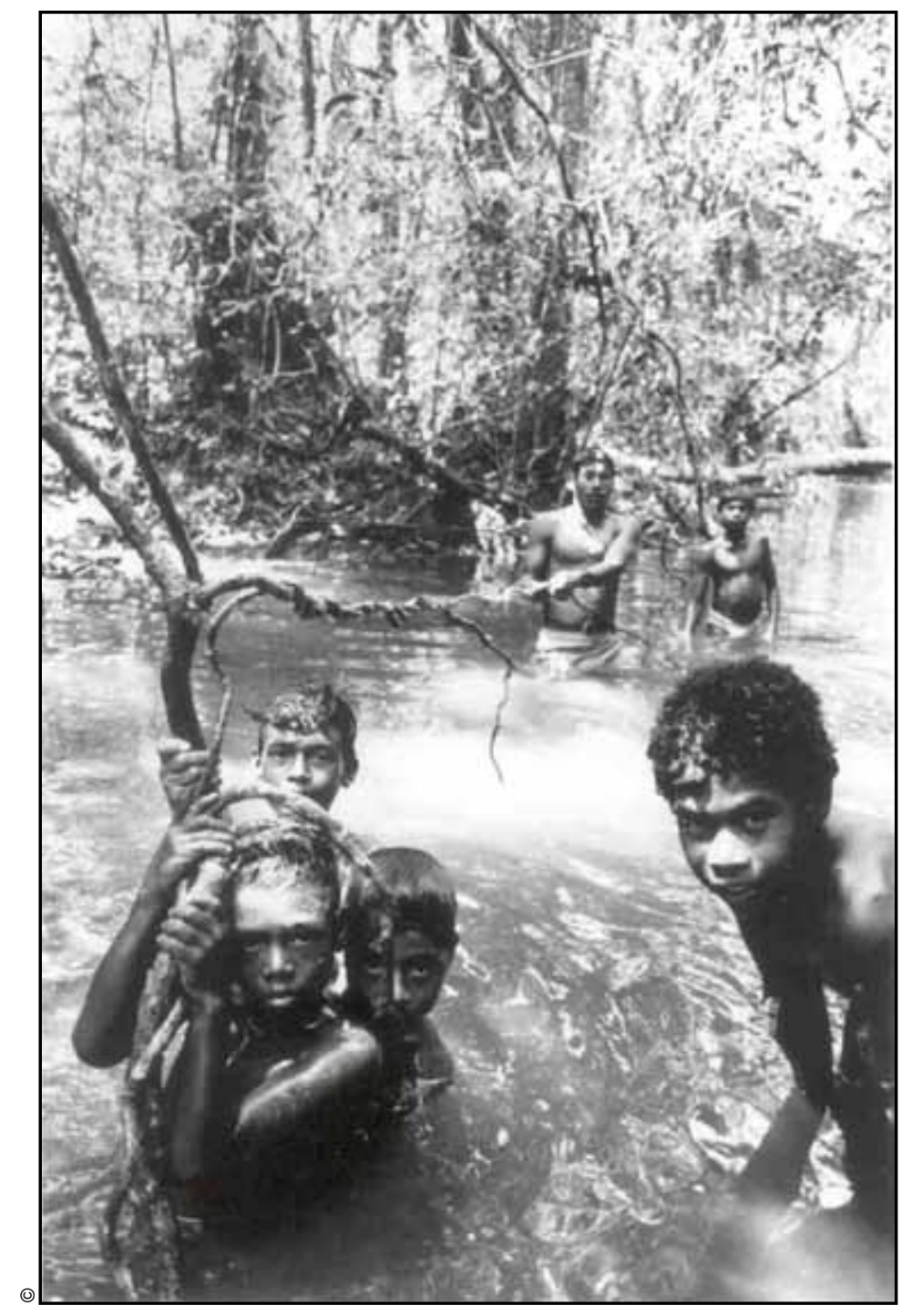

posible que la familia residente en Guadalupe sea descendiente de estos ulwas. El profesor Francisco Santiago entrevistó también a una señora de Mahogany Creek, en Bluefields, familiar de la familia de Guadalupe, que habla bien ulwa y que nos informó que hay más hablantes ulwa en esa comunidad.

Además, la familia de Makantakita nos informó de los siguientes lugares donde residen ulwas: Santa Rita, Mulukuku, Nazareth, Kulumbo, Waki Was, Breña, Ciudadela, Rio Blanco y Sihka. También se nos informó que en Tuma (en ulwa "tu lengua"), más arriba de Tumarín, se encuentran unas piedras con figuras dibujadas de una luna, un sol, una estrella y personas. Es posible que sean de interés arqueológico y que pudieran haber sido talladas por ulwas. Todos estos datos tendrán que ser confirmados en futuros viajes del CODIUL.
El CODIUL planea poder visitar estas comunidades para entablar contactos y entrevistar a los otros ulwas. Está pendiente igualmente la comparación lingüística de la variedad de ulwa hablada en Karawala y la variedad de estos hablantes entrevistados, ya que a primera vista se notaron diferencias interesantes.

Podemos decir que ambos proyectos aquí descritos fueron todo un éxito y contaron con el apoyo general de la comunidad de Karawala. Constituyen pequeños pasos hacia el objetivo final del CODIUL, que es restablecer el uso cotidiano del ulwa y la transmisión de la lengua a las nuevas generaciones. Estos esfuerzos responden a la idea que el prestigio de una lengua y el deseo de conocerla es un ingrediente indispensable para contrarrestar el cambio lingüístico que está teniendo lugar en la comunidad. A largo plazo, la creación de los DVDs responde al objetivo de incrementar el prestigio de la lengua ulwa en la comunidad. La creación de materiales en ulwa demuestra que la lengua ulwa es tan válida como cualquier otra para ser utilizada en cualquier ámbito, incluso en ámbitos más modernos como es la televisión o Internet.

El encuentro entre jóvenes de Karawala y jóvenes hablantes ulwa de otras comunidades puede servir de aliciente para el aprendizaje y el uso del ulwa por las nuevas generaciones, las cuales serán las encargadas de transmitir el ulwa a sus hijos en las próximas décadas. Por ejemplo, se podrían organizar intercambios o visitas de los jóvenes ulwa a Karawala donde se fomentara la utilización del ulwa entre los jóvenes de Karawala que están aprendiendo ulwa ahora en la escuela.

Una lengua constituye una forma única de expresar la realidad y, quizás lo que es más importante para la comunidad, una manera concreta de clasificar nuestro entorno. Una lengua encierra todo el conocimiento atesorado durante siglos por los antepasados de la comunidad, por lo que la pérdida de la lengua es sinónimo de la pérdida de este conocimiento en muchos casos. El CODIUL sigue trabajando para preservar la lengua ulwa, un patrimonio cultural de todos los nicaragüenses.

Aquí lengua en el sentido de parte del cuerpo. 


\section{Referencias}

CODIUL/ UYUTMUBAL, CIDCA, \& MIT. (1996). Diccionario preliminar del ulwa (sumo meridional).

Craig, C. G. (1992). Language shift and language death: the case of Rama in Nicaragua. International Journal of the Sociology of Language, (93), 11-26.

Green, T. (1999). A Lexicographic Study of Ulwa. Unplished P.h.D. Dissertation. Department of Linguistics and Philosophy, M.I.T.

Green, T., \& Hale, K. (1998). Ulwa, the language of Karawala, eastern Nicaragua: its position and prospects in modern Nicaragua. International Journal of the Sociology of Language, 1998(132), 185-202.

Hale, K. (1991). El ulwa, sumo meridional: ¿un idioma distinto? Wani, 11, 27-50.

Hale, K. (2001). Ulwa (Southern Sumu): the beginnings of a language research project. In P. Newman \& M. Ratliff, Linguistic fieldwork (pp. 76-101). Cambridge: Cambridge University Press.

Knight Julian, L., \& James Olegarios, M. M. (2008). Walangwas: Historial oral de la comunidad de Karawala. Bluefields, Nicaragua: URACCAN.

Koontz-Garboden, A. (2007). States, changes of state, and the monotonicity hypothesis.

Koontz-Garboden, A. (2009). Ulwa verb class morphology. International Journal of American Linguistics, 75(4), 453-512.

Koskinen, A. (1995). Language policy towards ethnic minorities in Northern Norway and on the Atlantic Coast of Nicaragua. International Journal of Educational Development, 15(3), 221-230.

Koskinen, A. (ed). (2007). El pueblo ulwa: Identidad y ambiente en un contexto multiétnico. Bluefields, Nicaragua: URACCAN.

Lewis, M. P. (2010). Ethnologue: Languages of the world (16th ed.). Dallas, Tex.: SIL International. Retrieved from http://www.ethnologue.com/.

Nettle, D., \& Romaine, S. (2000). Vanishing voices: the extinction of the world's languages. Oxford: Oxford University Press.

Salazar, O. (2007). Situación sociolingüística del pueblo ulwa en la comunidad de Karawala. In A. Koskinen, El pueblo ulwa: Identidad y ambiente en un contexto multiétnico (pp. 27-46). Bluefields, Nicaragua: URACCAN.

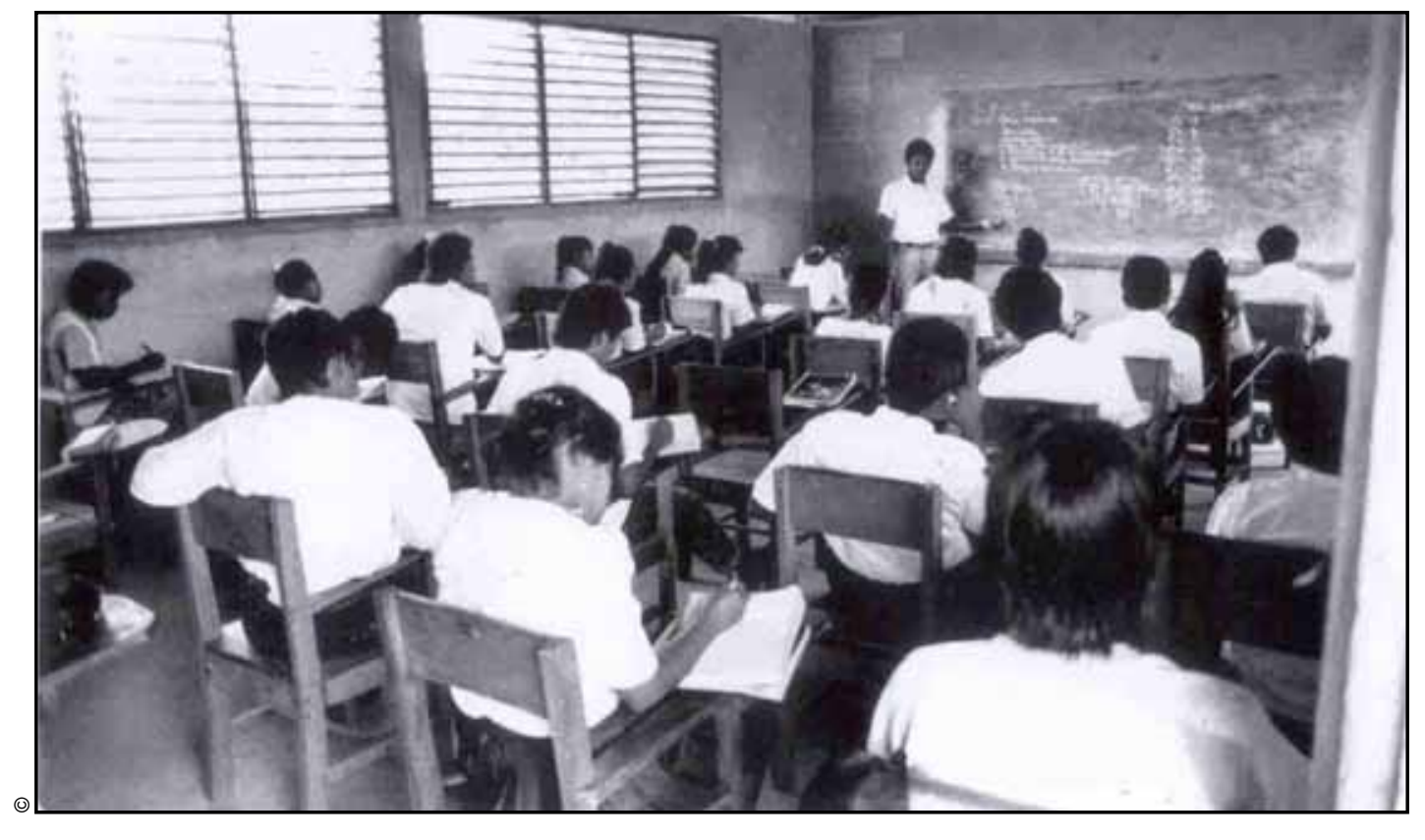

